\title{
Spatiotemporal analysis of rental bike data in Budapest
}

\author{
Eszter Kovács $^{a}$, Mátyás Gede ${ }^{\mathrm{a}, *}$ \\ ${ }^{a}$ Department of Cartography and Geoinformatics, ELTE Eötvös Loránd University, Eszter Kovács - eszterkovacs097@gmail.com, \\ Mátyás Gede-saman@map.elte.hu \\ * Corresponding author
}

Keywords: spatiotemporal analysis, bike rental service, isochrones

\begin{abstract}
:
The largest bike rental service of Budapest, MOL Bubi, started its operation in 2014. Since then it became an important supplement and alternative to driving and public transport in the city. This paper analyses bicycle usage data of year 2018.

The raw data included records of the starting and finishing location and time of each usage event (approx.. 500000 records in 2018), and anonymised metadata of the users. Data processing was carried out in QGIS, using its Spatialite database engine, similarly to the previous work of Gede, Varga (2019). In addition to simple temporal analysis (daily, weekly, monthly usage patterns) the data had been completed by the optimum routes between docker stations based on the freely available OpenRouteService routing engine (Figure 1, left). Comparing the actual timespan of a rental event with the duration of the optimum route between its endpoints made it possible to determine whether a bicycle was used to get directly from A to B or it was rather used to explore the city without having the actual GPS track of the ride. Creating isochrones map of the docker stations (Figure 1, right) also revealed the "bike rental coverage" of the city as most users never exceed the 30 minutes rental timespan because there is an extra cost beyond this time limit.
\end{abstract}
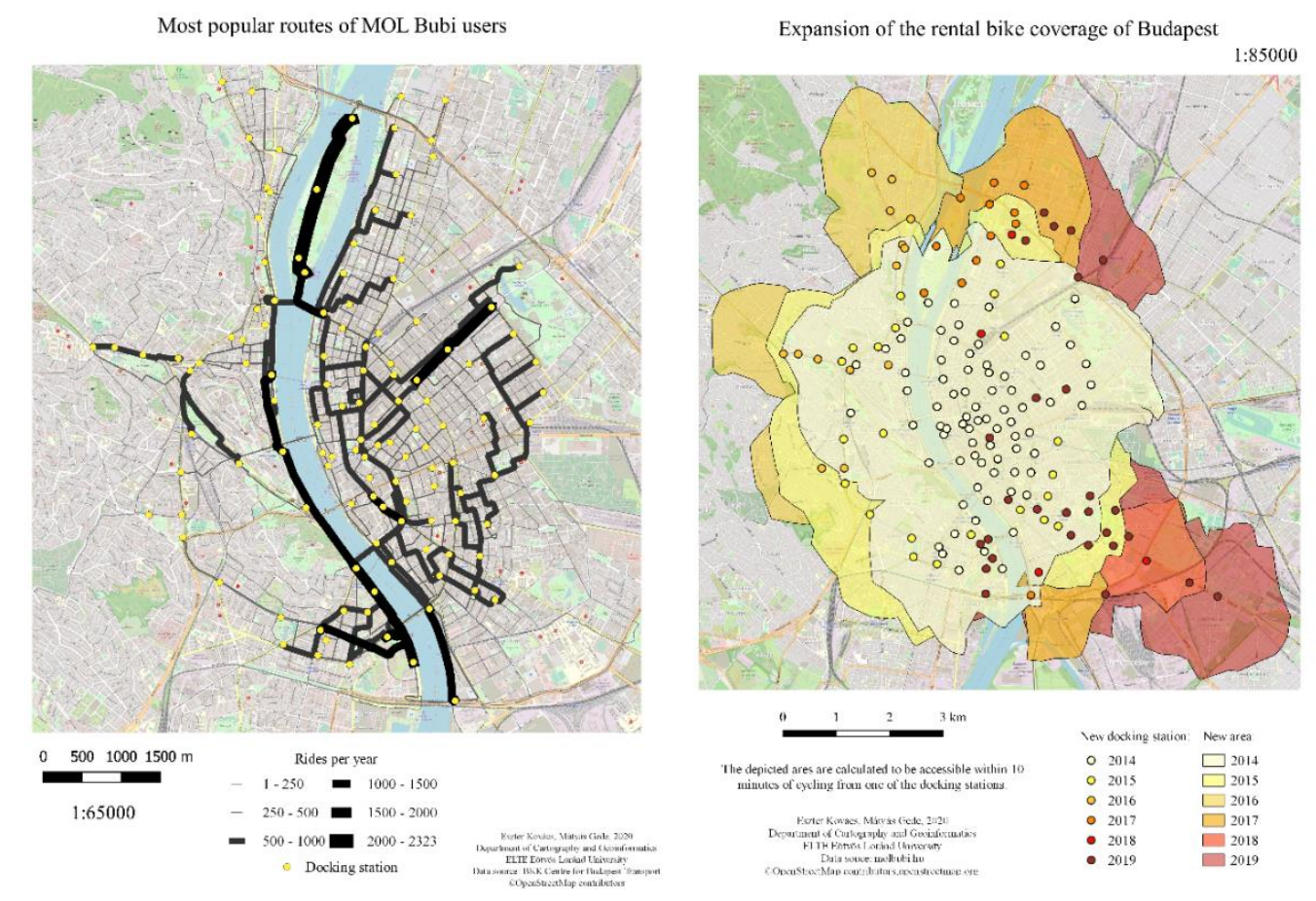

Figure 1. Most popular routes of MOL Bubi users (left) and expansion of the rental bike coverage of Budapest (right)

\section{References}

Gede, M. and Varga, L.: Spatiotemporal Analysis of Carpooling Data, Abstr. Int. Cartogr. Assoc., 1, 93, https://doi.org/10.5194/ica-abs-1-93-2019, 2019. 